\title{
Time Dependent Approach to Scattering from Impurities in a Crystal
}

\author{
Lawrence E. Thomas \\ Département de Physique Théorique, Section de Physique, Université de Genève, \\ Genève, Suisse
}

Received March 20; in revised form July 13, 1973

\begin{abstract}
A time dependent scattering theory for a quantum mechanical particle moving in an infinite, three dimensional crystal with impurity is given. It is shown that the Hamiltonian for the particle in the crystal without impurity has only absolutely continuous spectrum. The domain of the resulting wave operators is therefore the entire Hilbert space.
\end{abstract}

\section{Introduction}

In the time dependent approach to potential scattering theory, one endeavors to establish the existence and completeness of wave operators $\Omega_{ \pm}=\operatorname{s-lim}_{t \rightarrow \pm \infty} e^{i t H_{1}} e^{-i t H}$ acting in a Hilbert space $\mathscr{H}$, where $H$ is the Hamiltonian operator for the free evolution of the system and $H_{1}$ is the perturbed Hamiltonian operator for the system with interactions. Here completeness means that the ranges of $\Omega_{ \pm}$coincide with the absolutely continuous subspace of $\mathscr{H}$ with respect to $H_{1}$. The unitary scattering operator $S$ is then given simply by $S=\Omega_{+}^{\dagger} \Omega_{-}[1,2]$.

Mathematically rigorous results asserting the existence and completeness of $\Omega_{ \pm}$have been given in the particular case of single particle potential scattering in which $-H=\Delta$ is the Laplacian acting in $L^{2}\left(\mathbb{R}^{3}\right)$, and $H_{1}=H+W(\boldsymbol{x})$, where $W(\boldsymbol{x})$ is a short range potential, e.g. $W(\boldsymbol{x}) \in L^{1}\left(\mathbb{R}^{3}\right) \cap L^{2}\left(\mathbb{R}^{3}\right)[2-4]$. It is a natural question to ask whether these results may be modified to accommodate the situation in which both $H$ and $H_{1}$ are altered by the addition of a periodic potential $V(\boldsymbol{x})$, that is, where $H$ and $H_{1}$ are given by $H=-\Delta+V(\boldsymbol{x}), H_{1}=-\Delta+V(\boldsymbol{x})$ $+W(\boldsymbol{x})$ and $V(\boldsymbol{x})=V(\boldsymbol{x}+\boldsymbol{a})=V(\boldsymbol{x}+\boldsymbol{b})=V(\boldsymbol{x}+\boldsymbol{c})$ for three linearly independent vectors $\boldsymbol{a}, \boldsymbol{b}, \boldsymbol{c}$. The modification would constitute a theory of scattering from an impurity $W(\boldsymbol{x})$ in a crystal, with the role of free Hamiltonian played by $H=-\Delta+V(x)$ [5].

We show that such a modification is indeed possible assuming that $V(x)$ is square integrable over a unit cell (Theorem 3, Section III). In fact this modification is almost immediate except for one technical point: since the domain of the resulting wave operators is only the 
absolutely continuous subspace $\mathscr{H}_{\text {a.c. }}$ of $\mathscr{H}$ with respect to $H$, it is of course desirable that this domain be non-empty so that the scattering theory is non-trivial. Thus Section II gives a spectral characterization of $H=-\Delta+V(\boldsymbol{x})$ showing that $\mathscr{H}_{\text {a.c. }}=\mathscr{H}$, i.e. $H$ has no normalizable eigenfunctions, and the continuous spectrum of $H$ is absolutely continuous.

These spectral properties are not surprising. The non-existence of normalizable eigenfunctions in one dimension is well known from ordinary differential equation arguments, and the absolute continuity of the spectrum is not hard to deduce from the resolvent $[6,7]$. But a proof of these spectral properties becomes more difficult in higher dimensions. Since they are fundamental to the time dependent theory of scattering, the principal part of this article is devoted to a discussion of just these properties. For additional spectral properties see [8-10].

\section{Spectral Properties of Hamiltonians with Periodic Potentials}

It will be convenient to work in momentum space. Let $H$ be the self adjoint operator acting in $\mathscr{H}=L^{2}\left(\mathbb{R}^{3}\right)$ defined by

$$
(H \varphi)(\boldsymbol{p})=p^{2} \varphi(\boldsymbol{p})+\sum_{q \in \Gamma} v_{\boldsymbol{q}} \varphi(\boldsymbol{p}+\boldsymbol{q}), \quad \boldsymbol{p} \in \mathbb{R}^{3}
$$

where $\Gamma$ is the reciprocal lattice, and $v_{q}$ are the Fourier coefficients of the (real) potential $V(\boldsymbol{x})$. It is assumed that $\sum_{q \in \Gamma}\left|v_{\boldsymbol{q}}\right|^{2}=v^{2}$ is finite, which corresponds to $V(\boldsymbol{x})$ being square integrable over a unit cell in configuration space. (We follow the notation of [11].) Let $\boldsymbol{k}$ be any point in the first Brillouin zone B, and set $\Gamma_{k}=\left\{\boldsymbol{j} \in \mathbb{R}^{3} \mid \boldsymbol{j}=\boldsymbol{k}+\boldsymbol{q}, \boldsymbol{q} \in \Gamma\right\}$. Denote as $\ell_{k}^{2}$ the Hilbert space of square summable functions $\left\{\varphi^{k}\right\}$ defined on $\Gamma_{k}$ with norm $\left\langle\varphi^{k}, \varphi^{k}\right\rangle_{k}=\sum_{j \in \Gamma_{k}} \bar{\varphi}^{k}(\boldsymbol{j}) \varphi^{k}(\boldsymbol{j})$. We obtain a direct integral decomposition of $\mathscr{H}$ which we write $\mathscr{H}=\int_{B}^{\oplus} \ell_{k}^{2} d^{3} k$. As is well known, the point of this decomposition is that it reduces $H$; if $\varphi=\int_{B}^{\oplus} \varphi^{k} d^{3} k$, then

$$
H \varphi=\int_{B}^{\oplus} H(k) \varphi^{k} d^{3} k
$$

with $H(k)$ acting in $\ell_{k}^{2}$ defined by

$$
\begin{aligned}
\left(H(k) \varphi^{k}\right)(\boldsymbol{j}) & =\left((T(k)+V(k)) \varphi^{k}\right)(\boldsymbol{j})=j^{2} \varphi^{k}(\boldsymbol{j})+\sum_{q \in \boldsymbol{I}_{k}} v_{\boldsymbol{j}-\boldsymbol{q}} \varphi^{k}(\boldsymbol{q}), \\
\left(T(k) \varphi^{k}\right)(\boldsymbol{j}) & =j^{2} \varphi^{k}(\boldsymbol{j}), \quad \boldsymbol{j} \in \Gamma_{k} .
\end{aligned}
$$


A study of the spectral properties of $H$ is then effected by a study of those of $H(k)$.

In [11], it is shown that the operators $(z-T(k))^{-1}$ and $V(k)(z-T(k))^{-1}$ are Hilbert-Schmidt for $z$ not in the spectrum of $T(k)$. Also, the resolvent $R(z, H(k))=(z-H(k))^{-1}=(z-T(k))^{-1} \cdot\left(1-V(k)(z-T(k))^{-1}\right)^{-1}$ is Hilbert-Schmidt for $z$ not in the spectrum of $H(k)$. The spectrum of $H(k)$ consists of (real) isolated points of finite multiplicity. Given any $R \in \mathbb{R}$, only a finite number of eigenvalues of $H(k)$ are less than $R$.

Let $U_{k}: \ell_{k}^{2} \rightarrow \ell_{0}^{2} \boldsymbol{k} \in B$ be the unitary map defined by

$$
\left(U_{k} \varphi^{k}\right)(\boldsymbol{j})=\varphi^{k}(\boldsymbol{j}+\boldsymbol{k}), \quad \boldsymbol{j} \in \Gamma .
$$

Then we can define the operator $H^{k}(0)$ acting in $\ell_{0}^{2}$ to be

with

$$
H^{k}(0)=U_{k} H(k) U_{k}^{-1}=H(0)+2 \boldsymbol{k} \cdot \boldsymbol{P}+k^{2}
$$

$$
\left(P_{i} \varphi^{0}\right)(\boldsymbol{j})=j_{i} \varphi^{0}(\boldsymbol{j}), \quad i=1,2,3, \boldsymbol{j} \in \Gamma .
$$

$H^{k}(0)$ is thus unitarily equivalent to $H(k)$. Although $H^{k}(0)$ was defined for $\boldsymbol{k} \in B$, we can extend the definition of $H^{k}(0)$ in $\boldsymbol{k}$ throughout the complex plane $\mathbb{C}^{3}$ by the right hand side of (5). Because the domain $\mathscr{D}\left(H^{k}(0)\right)=\mathscr{D}(H(0))=\mathscr{D}(T(0))=\mathscr{D}^{0}$ is independent of $\boldsymbol{k}$ and $H^{k}(0) \varphi^{0}$ is a holomorphic function of $\boldsymbol{k}$ for $\varphi^{0} \in \mathscr{D}^{0}\left[H^{k}(0)\right.$ is a closed operator which is self adjoint for $\boldsymbol{k}$ real], it follows that $H^{k}(0)$ is a self adjoint type A holomorphic family in each of the variables $k_{i}, i=1,2,3$ ([2], pp. 375, 385). We elaborate on the analytic behavior of the eigenvalues of $H^{k}(0)$ as functions of $\boldsymbol{k}$. Assume that coordinates are chosen so that the $k_{3}$-direction is perpendicular to one of the faces of the Brillouin zone B.

Lemma 1. Let $k_{1}, k_{2}$ be held constant and real. Then each eigenvalue $\lambda_{n}(k)$ of $H^{k}(0)$ and its corresponding projection $P_{n}^{k}(0)$, regarded as functions of $k_{3}$, may be taken to be holomorphic in a neighborhood of the real axis. $\lambda_{n}(k)$ is not constant as a function of $k_{3} .(\{n\}$ is a numbering of the eigenvalues, including degeneracies.)

Proof. A proof of the holomorphy of the $\lambda_{n}$ 's is given in [8]. We remark that the holomorphy of the eigenvalues and projections is a consequence of $H^{k}(0)$ being a self adjoint type A holomorphic family with compact resolvent ([2], p. 392). Finally, suppose to the contrary that $\lambda_{n}(k)=\lambda_{n}$ is constant in $k_{3}$, for some $k_{1}, k_{2}$ and $n$. Let $T^{k}(0)$ acting in $\ell_{0}^{2}$ be defined by $T^{k}(0) \varphi^{0}=\left(T(0)+2 \boldsymbol{k} \cdot \boldsymbol{P}+k^{2}\right) \varphi^{0}, \varphi^{0} \in \mathscr{D}^{0}$. Set $D=\left\{k_{3} \in \mathbb{C} \mid\left(\lambda_{n}-T^{k}(0)\right)^{-1}\right.$ is bounded $\}$. Since

$$
\left(\lambda_{n}-H^{k}(0)\right)^{-1}=\left(\lambda_{n}-T^{k}(0)\right)^{-1}\left(1-V(0)\left(\lambda_{n}-T^{k}(0)\right)^{-1}\right)^{-1}
$$


is not bounded for $k_{3}$ in a neighborhood $N$ of the real line, 1 must be an eigenvalue of the compact operator $V(0)\left(\lambda_{n}-T^{k}(0)\right)^{-1}$ for $k_{3}$ in $N \cap D$ and hence throughout $D$ ([2], Theorem 1.9, p. 370). But this is impossible; for $\left|\operatorname{im} k_{3}\right|$ sufficiently large, and re $k_{3}$ so that inf $\mid$ re $k_{3}+j_{3} \mid>0$ $\boldsymbol{j} \in \Gamma$, we obtain $\left\|V(0)\left(\lambda_{n}-T^{k}(0)\right)^{-1}\right\|<1$. In particular, the HilbertSchmidt norm of this operator may be made arbitrarily small, $\left|\operatorname{im} k_{3}\right| \rightarrow \infty$. (See Lemma A-1 of the Appendix.)

Remark. Although the $\lambda_{n}$ 's can be chosen holomorphic in any one of the variables $k_{i}$, they will not in general be single valued as a function of all three variables $k_{i}$. Typically, there will be curves running through $\mathrm{B}$ such that $\lambda_{n}(k)$ will experience a discontinuity as $k$ passes in a loop about a curve. Hence the labeling $\{n\}$ of the eigenvalues will be discontinuous. For the sake of definiteness, choose $\lambda_{n}\left(k_{1}, k_{2}, k_{3}\right)$ to be an analytic continuation in $k_{3}$ of $\lambda_{n}\left(k_{1}, k_{2}, 0\right)$, where the $\lambda_{n}\left(k_{1}, k_{2}, 0\right)$ are ordered according to magnitude.

Lemma 2. Let $B_{\lambda}=\{\boldsymbol{k} \in B \mid \lambda$ is an eigenvalue of $H(k)\}$. Then $\mu\left(B_{\lambda}\right)=0$ where $\mu$ is Lebesgue measure in $B$.

Proof. Equivalently, $B_{\lambda}=\left\{\boldsymbol{k} \in B \mid \lambda\right.$ is an eigenvalue of $\left.H^{k}(0)\right\}$. Let $q \in \mathscr{C} B_{\lambda}$, the complement of $B_{\lambda}$ in $B$. For some $\varepsilon>0$, $\lambda$ will not be an eigenvalue of $H^{k}(0),|\boldsymbol{k}-\boldsymbol{q}|<\varepsilon$; hence $\mathscr{C} B_{\lambda}$ is open and $B_{\lambda}$ is closed. Thus $B_{\lambda}$ is measurable. Let $f(k)$ be its characteristic function. Then by Fubini's theorem ([12], p. 190), we have $\mu\left(B_{\lambda}\right)=\int_{B} f(k) d k_{3} d k_{1} d k_{2}$ so that $\mu\left(B_{\lambda}\right)=0$ if $\int f(k) d k_{3}=F\left(k_{1}, k_{2}\right)=0$. But $F\left(k_{1}, k_{2}\right)=\int_{S\left(k_{1}, k_{2}\right)} d k_{3}$ where $S\left(k_{1}, k_{2}\right)$ $=\left\{k_{3} \mid \lambda\right.$ is an eigenvalue of $\left.H^{k}(0)\right\}=\left\{k_{3} \mid \lambda-\lambda_{n}(k)=0\right.$ for some $\left.n\right\}$. (Only a finite number of $\lambda_{n}$ 's need be considered since for $n$ sufficiently large $\lambda_{n}>\lambda+1$. See Lemma A-2 of the appendix.) Thus by Lemma 1, $S\left(k_{1}, k_{2}\right)$ is the zero set of a finite number of non-constant holomorphic functions in $k_{3}$, which clearly has measure zero.

Although the proof that $H$ has no normalizable eigenfunctions is contained implicitly in the proof of Theorem 2 below, it is easy to give a separate proof of that fact here.

Theorem 1. The spectrum of $H$ is pure continuous.

$$
\text { Proof. Let }(H-\lambda) \varphi=\int_{B}^{\oplus}(H(k)-\lambda) \varphi^{k} d^{3} k=0 \text {. Then }(H(k)-\lambda) \varphi^{k}=0
$$

almost everywhere in $B . \varphi^{k}$ vanishes almost everywhere for $\boldsymbol{k} \in \mathscr{C} B_{\lambda}$. On the other hand, by Lemma $2 \mu\left(B_{\lambda}\right)=0$ implying $\varphi^{k}=0$ a.e. in $B$.

We next prove that the continuous spectrum of $H$ contains no singular continuous part. 
Theorem 2. The spectrum of $H$ is absolutely continuous.

Proof. It suffices to show that $\left\langle\varphi(z-H)^{-1} \varphi\right\rangle$ has continuous boundary values in $z$ as $z$ approaches any bounded interval $I$ of the real axis, for $\varphi$ in some dense set $\mathscr{D}_{I} \subset \mathscr{H}$. In this case, the quantity $\frac{1}{2 \pi i} \lim _{\varepsilon \rightarrow 0}\left\{\left\langle\varphi(\lambda-i \varepsilon-H)^{-1} \varphi\right\rangle-\left\langle\varphi(\lambda+i \varepsilon-H)^{-1} \varphi\right\rangle\right\}$ is continuous in $\lambda \in \mathbb{R}$. But this limit is just the Radon-Nikodym derivative of $\langle\varphi E(\lambda) \varphi\rangle$ with respect to Lebesgue measure, where $E(\lambda)$ is the spectral family of $H$. This shows that the spectral family $E(\lambda)$ is absolutely continuous in $I$ and, since $I$ is arbitrary, in the entire real line ([2], Chapter 10).

Let $\mathscr{D}_{0}$ consist of finite linear combinations of the function $\varphi=e^{-p_{1}{ }^{2}-p_{2}{ }^{2}-p_{3}{ }^{2}}$ and its translates in $\mathscr{H}=L^{2}\left(\mathbb{R}^{3}\right) . \mathscr{D}_{0}$ is dense in $\mathscr{H}$. It is clear that $\varphi_{\mid \Gamma_{k}} \in \ell^{2}{ }_{k}$ if $\varphi \in \mathscr{D}_{0}$. If $\boldsymbol{q} \in \mathbb{C}^{3}$ and $\varphi \in \mathscr{D}_{0}, \varphi_{q}(\boldsymbol{p})=\varphi(\boldsymbol{q}+\boldsymbol{p})$ $\in L^{2}\left(\mathbb{R}^{3}\right)$ and $\varphi_{q}^{k}=\varphi(\boldsymbol{p}+\boldsymbol{q})_{\mid \Gamma_{k}} \in \ell_{k}^{2}$. Note also that if $\psi^{k} \in \ell_{k}^{2},\left\langle\psi^{k}, \varphi_{q}^{k}\right\rangle_{k}$ will be holomorphic in $q \in \mathbb{C}^{3}$. The function

$$
\begin{aligned}
\left\langle\varphi^{k}, P_{n}(k) \varphi^{k}\right\rangle_{k} & =\left\langle U_{k} \varphi^{k}, U_{k} P_{n}(k) U_{k}{ }^{-1} U_{k} \varphi^{k}\right\rangle_{0} \\
& =\left\langle U_{k} \varphi^{k}, P_{n}{ }^{k}(0), U_{k} \varphi^{k}\right\rangle_{0} \varphi \in \mathscr{D}_{0},
\end{aligned}
$$

regarded as a function of $k_{3}$, will have a holomorphic continuation in a neighborhood of the real axis since both $P_{n}^{k}(0)$ and $U_{k} \varphi^{k}$ are holomorphic.

Next we define $\mathscr{D}_{I}$. Let $I$ be a bounded interval. There exists a finite $N>0$ such that if $n>N$, dist $\left(\lambda_{n}(k), I\right)>1$ for all $\boldsymbol{k} \in B$. (See Lemma A-2 of the Appendix.) Then we set $\mathscr{D}_{I}=F_{I} \mathscr{D}_{0}$ where $F_{I}$ is a bounded positive symmetric operator defined by

$$
F_{I} \varphi=\int_{B}^{\oplus} \frac{g(k)}{N\left(k_{1}, k_{2}\right)}\left(\prod_{i=1}^{N} \frac{\partial}{\partial k_{3}} \lambda_{i}(k)\right)^{2} \varphi^{k} d^{3} k
$$

where $g(k)$ is a $C^{2}$ function, $0 \leqq g \leqq 1$, which is non-zero at every interior point of $B$ and vanishes on the boundary $\partial B . N\left(k_{1}, k_{2}\right)$ is just a normalization defined by

$$
\begin{aligned}
N\left(k_{1}, k_{2}\right)= & \prod_{n=1}^{N}\left(1+\sum_{i=0}^{3} \sup _{\substack{k_{3} \\
k \in B}}\left|\left(\frac{\partial}{\partial k_{3}}\right)^{i} \lambda_{n}\right|+\sup _{\substack{k_{3} \\
k \in B}}\left\|\frac{\partial}{\partial k_{3}} P_{n}^{k}(0)\right\|\right. \\
& \left.+\sup _{\substack{k_{3} \\
k \in B}}\left\|\frac{\partial^{2}}{\partial k_{3}{ }^{2}} P_{n}{ }^{k}(0)\right\|^{2}\right) .
\end{aligned}
$$

$N\left(k_{1}, k_{2}\right)^{-1}$ will be piecewise continuous and non-vanishing in $B$. Since $g(k)\left[\prod_{i=1}^{N} \frac{\partial}{\partial k_{3}} \lambda_{i}\right]^{2}$ vanishes only on a set of measure zero, $F_{I} \varphi \neq 0$ for 
$\varphi \neq 0$. The normalization insures that $F_{I}$ is bounded. Finally, if $\psi \neq 0$ is in $\mathscr{H},\left\langle\psi, F_{I} \varphi\right\rangle=\left\langle F_{I} \psi, \varphi\right\rangle \neq 0$ for some $\varphi \in \mathscr{D}_{0}$ which implies $\mathscr{D}_{I}=F_{I} \mathscr{D}_{0}$ is dense in $\mathscr{H}$.

Let $z$ lie in the resolvent set of $H$. For any $\varphi \in \mathscr{H}$, we have

$$
\begin{aligned}
\left\langle\varphi(z-H)^{-1} \varphi\right\rangle & =\int_{B} \sum_{n} \frac{\left\langle\varphi^{k} P_{n}(k) \varphi^{k}\right\rangle}{z-\lambda_{n}(k)} d^{3} k \\
& =\sum_{n=1}^{N} \int_{B} \frac{\left\langle\varphi^{k} P_{n}(k) \varphi^{k}\right\rangle}{z-\lambda_{n}(k)} d^{3} k+\int_{B} \sum_{n>N} \frac{\left\langle\varphi^{k} P_{n}(k) \varphi^{k}\right\rangle}{z-\lambda_{n}(k)} d^{3} k .
\end{aligned}
$$

The latter term will be a bounded analytic function of $z$ in a neighborhood of $I$, since dist $\left(\lambda_{n}, I\right)>1$ for $n>N$. It only remains to show that the first term has continuous boundary values in $z$ for $\varphi=F_{I} \varphi_{0} \in \mathscr{D}_{I}$, i.e. $\varphi_{0} \in \mathscr{D}_{0}$. But the first term is just

$$
\begin{aligned}
J(z)= & \sum_{n}^{N} \int_{B} d^{3} k \frac{\left\langle\varphi^{k} P_{n}^{(k)} \varphi^{k}\right\rangle}{z-\lambda_{n}(k)} \\
= & \sum_{n}^{N} \int_{B} \frac{d^{3} k g^{2}(k)}{N^{2}\left(k_{1}, k_{2}\right)}\left(\prod_{i=1}^{N} \frac{\partial}{\partial k_{3}} \lambda_{i}\right)^{4} \frac{\left\langle\varphi_{0}^{k} P_{n}(k) \varphi_{0}^{k}\right\rangle}{z-\lambda_{n}(k)} \\
= & \sum_{n}^{N} \int_{B} \frac{d^{3} k}{N^{2}\left(k_{1}, k_{2}\right)} \ln \left(z-\lambda_{n}(k)\right) \frac{\partial}{\partial k_{3}} \\
& \cdot\left\{g^{2}(k)\left(\prod_{i \neq n}^{N} \frac{\partial}{\partial k_{3}} \lambda_{i}\right)^{4}\left(\frac{\partial}{\partial k_{3}} \lambda_{n}\right)^{3}\left\langle\varphi_{0}{ }^{k} P_{n}(k) \varphi_{0}{ }^{k}\right\rangle\right\}
\end{aligned}
$$

where we have integrated by parts in $k_{3}$, and used the fact that $g=0$ on $\partial B$. Integrating by parts once more in $k_{3}$, we obtain

$$
\begin{aligned}
J(z)= & \sum_{n}^{N} \int_{B} \frac{d^{3} k}{N^{2}\left(k_{1}, k_{2}\right)}\left(z-\lambda_{n}\right) \frac{\partial}{\partial k_{3}} \\
& \cdot\left\{\ln \left(z-\lambda_{n}\right)\left[\left(\frac{\partial}{\partial k_{3}} G_{n}\right)\left(\frac{\partial}{\partial k_{3}} \lambda_{n}\right)^{2}+3 G_{n}\left(\frac{\partial}{\partial k_{3}} \lambda_{n}\right)\left(\frac{\partial^{2}}{\partial k_{3}^{2}} \lambda_{n}\right)\right]\right\} \\
= & \sum_{n}^{N} \int_{B} \frac{d^{3} k}{N^{2}\left(k_{1}, k_{2}\right)}\left\{-\left(\frac{\partial}{\partial k_{3}} \lambda_{n}\right)+\left(z-\lambda_{n}\right) \ln \left(z-\lambda_{n}\right) \frac{\partial}{\partial k_{3}}\right\} \\
& \cdot\left\{\left(\frac{\partial}{\partial k_{3}} G_{n}\right)\left(\frac{\partial}{\partial k_{3}} \lambda_{n}\right)^{2}+3 G_{n}\left(\frac{\partial}{\partial k_{3}} \lambda_{n}\right)\left(\frac{\partial^{2}}{\partial k_{3}^{2}} \lambda_{n}\right)\right\}
\end{aligned}
$$

where

$$
G_{n}(k)=g^{2}(k)\left(\prod_{i \neq n}^{N} \frac{\partial}{\partial k_{3}} \lambda_{i}(k)\right)^{4}\left\langle\varphi_{0}{ }^{k} P_{n}(k) \varphi_{0}{ }^{k}\right\rangle .
$$


In this form it is clear that $J(z)$ will have continuous boundary values as $z \rightarrow I$. The normalization function was constructed to keep the integrand of the right hand side of (10) bounded. Hence we have the theorem.

\section{Scattering Theory}

Let $H_{w}=-\Delta+V(\boldsymbol{x})+W(\boldsymbol{x})$ act in $\mathscr{H}=L^{2}\left(\mathbb{R}^{3}\right)$ with $V(\boldsymbol{x})$ again a real periodic function of $\boldsymbol{x}$, square integrable over a unit cell, and $W(\boldsymbol{x})$ a real valued function of $\boldsymbol{x}$ satisfying the conditions of the theorem below. $W(\boldsymbol{x})$ is just the potential due to the impurity. $H$ is the "free" Hamiltonian, $H=-\Delta+V(\boldsymbol{x})$.

Theorem 3. Let $W$ be relatively compact with respect to $-\Delta$ and assume $|W|^{\frac{1}{2}}(1-\Delta)^{-1}$ is Hilbert-Schmidt. Then the wave operators $\Omega_{ \pm}\left(H_{w}, H\right)$ exist and are complete, i.e. the range of $\Omega_{ \pm}$is the absolutely continuous subspace with respect to $H_{w}$. The domain of $\Omega_{ \pm}$is $\mathscr{H}$.

Proof. Let $\lambda$ be a point below the spectrum of $H$. We have in the momentum representation that

$(1-\Delta)(\lambda-H)^{-1}=\int_{B}^{\oplus}(1+T(k))(\lambda-T(k))^{-1}\left(1-V(k)(\lambda-T(k))^{-1}\right)^{-1} d^{3} k$

is bounded. Therefore $W(\lambda-H)^{-1}=W(1-\Delta)^{-1}(1-\Delta)(\lambda-H)^{-1}$ is compact. Similarly $|W|^{\frac{1}{2}}(\lambda-H)^{-1}$ is Hilbert-Schmidt. By ([2], Theorem 4.9, p. 545) the wave operators exist and are complete. The domain of $\Omega_{+}$is the absolutely continuous subspace of $\mathscr{H}$ with respect to $H$, hence by Theorems 1 and 2, all of $\mathscr{H}$.

Remark. Perturbations satisfying Theorem 3 include $W(x) \in L^{1}\left(\mathbb{R}^{3}\right)$ $\cap L^{2}\left(\mathbb{R}^{2}\right)([2]$, p. 546). A theorem for such perturbations has been given by Lenahan [12], but with the periodic potential $\beta V(\boldsymbol{x})$ assumed bounded, and the coupling constant $\beta$ not assuming supposed exceptional values. Results on existence of wave operators have been shown for a broader class of perturbations $W(\boldsymbol{x})$ [12]. These results can probably be extended further $[3,4]$, but the main point here has been to establish the appropriate spectral properties for the unperturbed Hamiltonian $H$.

\section{Appendix}

We first prove a lemma needed for Lemma 1 of Section II.

Lemma A-1. For appropriate choice of re $k_{3}$, the Hilbert-Schmidt norm of $V(0)\left(\lambda-T^{k}(0)\right)^{-1}$ tends to zero, $\left|\mathrm{im} k_{3}\right|$ tending towards $\infty$. 
Proof. The Hilbert-Schmidt norm $S(k)$ is given by

$$
\begin{aligned}
S(k) & =\sum_{p, q \in \Gamma} v_{\boldsymbol{p}-\boldsymbol{q}} v^{*}{ }_{\boldsymbol{p}-\boldsymbol{q}}\left(\lambda-(\boldsymbol{q}+\boldsymbol{k})^{2}\right)^{-1}\left(\lambda-\left(\boldsymbol{q}+\boldsymbol{k}^{*}\right)^{2}\right)^{-1} \\
& =v^{2} \sum_{q \in \Gamma}\left(\left(\lambda-(\boldsymbol{q}+\mathrm{re} \boldsymbol{k})^{2}+(\operatorname{im} k)^{2}\right)^{2}+4(\operatorname{im} \boldsymbol{k} \cdot(\boldsymbol{q}+\mathrm{re} \boldsymbol{k}))^{2}\right)^{-1}, \quad \boldsymbol{q} \in \Gamma
\end{aligned}
$$

where $\boldsymbol{k}$ points in the $k_{3}$-direction. Fix re $\boldsymbol{k}$ so that $\mid q_{3}+$ re $k_{3} \mid$ does not vanish, $\boldsymbol{q} \in \Gamma$. (Recall that the $k_{3}$-direction was chosen perpendicular to a Brillouin zone face.) Then for some finite $M>0$ independent of im $k_{3}$,

$$
\begin{aligned}
& \left(\left(\lambda-q^{2}+(\operatorname{im} k)^{2}\right)^{2}+4(\operatorname{im} \boldsymbol{k} \cdot \boldsymbol{q})^{2}\right)^{-1} \\
& \quad \leqq M\left(\left(q^{2}-(\operatorname{im} k)^{2}\right)^{2}+4(\operatorname{im} \boldsymbol{k} \cdot \boldsymbol{q})^{2}+(\operatorname{im} k)^{2}\right)^{-1}, \quad \boldsymbol{q} \in \Gamma_{\mathrm{re} k} .
\end{aligned}
$$

But this latter term is in turn uniformly bounded in $\boldsymbol{q} \in \Gamma_{\mathrm{re} k}$ and $|\operatorname{im} \boldsymbol{k}| \geqq 1$ by $N\left(\left((\boldsymbol{q}+\boldsymbol{r})^{2}-(\operatorname{im} \boldsymbol{k})^{2}\right)^{2}+4(\operatorname{im} \boldsymbol{k} \cdot(\boldsymbol{q}+\boldsymbol{r}))^{2}+(\operatorname{im} \boldsymbol{k})^{2}\right)^{-1}$ for some finite $N>0, r \in B$. In short, for this choice of re $k_{3}$ and $\left|\operatorname{im} k_{3}\right| \geqq 1$,

$$
\begin{aligned}
S(k) & \leqq \mathrm{const} \int_{\mathbb{R}^{3}} d^{3} p\left(\left(p^{2}-(\operatorname{im} k)^{2}\right)^{2}+4(\boldsymbol{p} \cdot \operatorname{im} \boldsymbol{k})^{2}+(\operatorname{im} k)^{2}\right)^{-1} \\
& =\mathrm{const} \int_{-1}^{1} d \mu \int_{0}^{\infty} p^{2} d p\left(\left(p^{2}-(\operatorname{im} k)^{2}\right)^{2}+4 p^{2}(\operatorname{im} k)^{2} \mu^{2}+(\operatorname{im} k)^{2}\right)^{-1}
\end{aligned}
$$

in spherical coordinates. The $p$ integration can be done explicitly by making the substitution of variables $x=p^{2}$, extending the contour of integration to $(-\infty, \infty)$ (the integrand will be pure imaginary $x<0$ ), and then performing the integral by contour integration techniques. One obtains

$S(k) \leqq$ const re $\int_{-1}^{1} d \mu \frac{\left((\operatorname{im} k)^{2}\left(1-2 \mu^{2}\right)+i\left((\operatorname{im} k)^{2}+4(\operatorname{im} k)^{4}\left(\mu^{2}-\mu^{4}\right)\right)^{\frac{1}{2}}\right)^{\frac{1}{2}}}{\left((\operatorname{im} k)^{2}+4(\operatorname{im} k)^{4}\left(\mu^{2}-\mu^{4}\right)\right)^{\frac{1}{2}}}$.

The integrand of the right hand side is uniformly bounded and goes to zero $|\operatorname{im} k| \rightarrow \infty$ for almost all $\mu$. Hence by the Lebesgue dominated convergence theorem ([13], p. 151), $S(k) \rightarrow 0|\operatorname{im} k| \rightarrow \infty$.

Finally, we prove a remark made in the proof of absolute continuity for the spectrum of $H$. (See the remark following the proof of Lemma 1, regarding the numbering of the eigenvalues $\lambda_{n}(k)$.)

Lemma A-2. Given any $\lambda>0$, there exists an $N$ such that if $n>N$, $\lambda_{n}(k) \geqq \lambda$.

Proof. The proof of this lemma reduces to showing that if $N$ is chosen large enough, a continuation $\tilde{\lambda}_{n}(k)$ of $\lambda_{n}(0)$ to $\boldsymbol{k}_{0} \in B$ along a straight line $\ell$ in $B$ satisfies $\tilde{\lambda}_{n}(k) \geqq \lambda, n>N, \boldsymbol{k} \in \ell$. We have an estimate of the form $\left\|\sum_{i}^{3} \hat{\ell}_{i} \frac{\partial}{\partial k_{i}} H^{k}(0) \varphi^{0}\right\| \leqq a(b)\left\|\varphi^{0}\right\|+b\left\|H^{k}(0) \varphi^{0}\right\| \varphi^{0} \in \ell_{0}^{2}, \boldsymbol{k} \in B$ and where 
$b>0$ may be taken arbitrarily small [11]. But this estimate implies that $\left|\tilde{\lambda}_{n}(k)-\lambda_{n}(0)\right| \leqq \frac{1}{b}\left(a(b)+b \lambda_{n}(0)\right)\left(e^{b\left|k_{0}\right|}-1\right)([2]$, Theorem 3.6, p. 391). Choose $b$ so that $\left(e^{b d}-1\right)=\frac{1}{2}$ where $d=\sup _{k \in B}|\boldsymbol{k}|$. Then $\left|\tilde{\lambda}_{n}(k)-\lambda_{n}(0)\right|$ $\leqq \frac{1}{2} \frac{a(b)}{b}+\frac{1}{2} \lambda_{n}(0)$. But if $N$ is large enough, i.e. $\lambda_{n}(0)$ large enough, it is clear that $\lambda_{n}(k) \geqq \lambda$.

\section{References}

1. Jauch, J. M.: Helv. Phys. Acta 31, 127 (1958)

2. Kato, T.: Perturbation theory for linear operators. Chaps. 7, 10. New York: Springer Inc. 1966

3. Lavine, R.: Indiana Univ. Math. J. 21, 643 (1972)

4. Lavine, R.: J. Functional Anal. 12, 30 (1973)

5. Ziman,J.M.: Electrons and phonons. Chap. VI. London: Oxford University Press 1960

6. Titchmarsh,E.C.: Eigenfunction expansions, Part II. Chap. XXI. London: Oxford University Press 1958

7. Dunford, N., Schwartz, J. T.: Linear operators, Part II. Chap. XIII, Sec. 7. New York: Interscience Publishers Inc. 1963

8. Odeh,F., Keller, J.: J. Math. Phys. 5, 1499 (1964)

9. Scharf, G.: Helv. Phys. Acta 39, 556 (1966)

10. Eastham, M.S.P.: Proc. Roy. Soc. Edinburgh 69 A, 125 (1971)

11. Avron,J., Grossmann, A., Rodriguez, R.: Hamiltonians in the one electron theory of solids I, (preprint, Centre de Physique Théorique, C.N.R.S., Marseille 1972).

12. Lenahan, T.A.: On quantum mechanical scattering in periodic media. Ph.D. Thesis in Applied Mathematics, Univ. of Pennsylvania, (1970), Chap. IV, (unpublished)

13. Dunford, N., Schwartz, J.T.: Linear operators, Part I. New York: Interscience Publishers, Inc. 1958

L. E. Thomas

Département de Physique Théorique

Section de Physique

Université de Genève

$\mathrm{CH}-1211$ Genève, Switzerland 
